\title{
LISTERIA SPP., COLIFORMES TOTAIS E FECAIS E E.COLI NO LEITE CRU E PASTEURIZADO DE UMA INDÚSTRIA DE LATICÍNIOS, NO ESTADO DA PARAÍBA (BRASIL)1
}

\author{
Raïssa Mayer Ramalho CATÃO ${ }^{2, *}$, Beatriz Susana Ovruski de CEBALLOS ${ }^{3}$
}

\begin{abstract}
RESUMO
Investigou-se a qualidade microbiológica do leite in natura e na linha de produção (leite recém-pasteurizado e leite ensacado), de uma usina de beneficiamento em Campina Grande-PB. Foi pesquisada a presença de Listeria spp. e sua diversidade de espécies, os níveis de coliformes totais (CT), coliformes fecais (CF) e Escherichia coli. Analisou-se um total de 75 amostras de leite, sendo 45 de leite cru, 15 de leite recém-pasteurizado e 15 de leite ensacado. Os resultados foram reunidos em dois grupos segundo o período de monitoramento: antes e após mudanças no processo de higienização da usina. Foi evidenciada elevada contaminação nas amostras de leite cru nas duas épocas. Na primeira (março-abril/1998), todas as amostras de leite beneficiado estiveram fora dos padrões da legislação vigente para CT e CF; na segunda (maio-agosto/1998), houve acentuada redução dos níveis destas bactérias indicadoras, porém as melhorias na higienização não foram suficientes para solucionar este problema, visto que $11,1 \%$ das amostras recém-pasteurizadas estavam fora dos padrões para CT e $33,3 \%$ para CF. Das amostras ensacadas, $22,2 \%$ estavam fora dos padrões para CT e $44,4 \%$ para CF. Comparando-se os resultados de CT, CF, e E.coli nas amostras de leite recém-pasteurizado e não ensacado com as amostras de leite ensacado, foi verificado que as amostras após serem pasteurizadas e ensacadas apresentaram valores de CT e CF levemente mais elevados, sugerindo contaminação durante o processo de ensacamento ou falhas na armazenagem. Observou-se que $33(73,3 \%)$ das amostras de leite cru e 9 (30\%) das de leite pasteurizado estavam contaminadas com Listeria spp., sendo identificadas $L$. monocytogenes em 17 (51,5\%) amostras de leite cru e em 9 (100\%) de leite beneficiado (4 recém-pasteurizadas e 5 ensacadas). Em relação à diversidade de espécies, nas amostras de leite cru foram encontradas: $L$. monocytogenes $(66,6 \%)$, L. innocua $(25,3 \%)$, L. ivanovii $(3,9 \%)$, L. welshimeri $(2,5 \%)$ e L. grayi $(1,5 \%)$. Nas amostras de leite pasteurizado isolaram-se: $L$. monocyogenes e $L$. innocua. O conjunto dos resultados evidenciou deficiências higiênico-sanitárias no leite in natura e ao longo do processo de produção, resultando em porcentagens elevadas de amostras que ultrapassaram os valores padrões de CT e CF além de apresentarem-se contaminadas por Listeria spp., com predominância de L. monocytogenes, sugerindo a existência de uma relação direta entre os altos índices de coliformes e a presença de Listeria spp.
\end{abstract}

Palavras-chave: L. monocytogenes; leite; contaminação.

\footnotetext{
${ }^{1}$ Recebido para publicação em 10/01/00. Aceito para publicação em 24/08/01. Parte da dissertação de mestrado apresentada por R.M.R. Catão, ao Curso de Mestrado em Ciência e Tecnologia de Alimentos da Universidade Federal da Paraíba-UFPB.

${ }^{2}$ Departamento de Farmácia e Biologia da Universidade Estadual da Paraíba-UEPB, Campus I - Campina Grande-PB.Av. Floriano Peixoto, 718 - Campina Grande-PB. CEP 58100-001. ${ }^{3}$ Departamento de Tecnologia Química e de Alimentos do Centro de Tecnologia da Universidade Estadual da Paraíba-UFPB Campus I - João Pessoa-PB e DEC/CCT/UFPB - Campus I Campina Grande -PB.

${ }^{*}$ A quem a correspondência deve ser enviada.
}

\begin{abstract}
SUMMARY
LISTERIA SPP., TOTAL COLIFORM, FECAL COLIFORM AND E.COLI ON RAW AND PASTEURIZED MILK ON A DAIRY INDUSTRY, (PARAÍBA, BRAZIL). The microbiological quality of raw and pasteurized milk was studied along its line production in a dairy factory in Paraíba State, Brazil. Listeria monocytogenes was frequent found in raw milk and the levels of total coliforms (TC), fecal coliforms (FC) and Escherichia coli, were high in the same samples. The results of 75 milk studied samples (45 raw milk, 15 recently pasteurized and 15 sacked), were distributed in two clusters, according its collected period (before and after changes in factory's hygienic process). Both raw samples groups showed high contamination. In the first period (March-April/1998), all the pasteurized milk were out of total and fecal coliforms standards. During the second period (May-August/1998), there was a significant reduction of coliforms levels, however the progress in hygienic conditions were not enough for eliminate the contamination: $11.1 \%$ of recent pasteurized milk samples and $22.2 \%$ of sacked milk samples were out of TC and also $33.3 \%$ and $44.4 \%$ recent pasteurized and sacked milk samples. Sacked milk presented more TC and FC than recent pasteurized milk, suggesting contamination after pasteurization or failure at storage. Listeria spp. was found in 33 raw milk samples $(73.3 \%)$ and in $9(30 \%)$ of pasteurized milk samples. L. monocytogenes was isolated in $17(51.5 \%)$ raw milk samples and in $9(100 \%)$ pasteurized milk samples (4 recent-pasteurized and 5 sacked). The Listeria spp. diversity was: L. monocytogenes $(66.6 \%)$, L. innocua $(25.3 \%)$, L. ivanovii (3.9\%) L. welshimeri $(2.5 \%)$ and L. grayi $(1.5 \%)$. In the pasteurized milk was isolated only: L. monocytogenes and $L$. innocua. The results suggest a direct relation between high levels of coliforms and presence of Listeria spp.
\end{abstract}

Keywords: L. monocytogenes; milk; contamination.

\section{1 - INTRODUÇÃO}

Durante o processo de produção, elaboração, transporte, armazenamento e distribuição, qualquer alimento está sujeito à contaminação por substâncias tóxicas ou por bactérias patogênicas, vírus e parasitos. O leite, devido à sua riqueza nutritiva, constitui um excelente meio de cultura para o desenvolvimento de diversos microrganismos, sendo veículo de transmissão de importantes zoonoses para o homem [12].

No Brasil, de modo geral, o leite é obtido sob condições higiênico-sanitárias deficientes, e em conseqüência, apresenta elevados números de microrganismos, o que constitui um risco à saúde da população, principalmente quando consumido sem tratamento térmico [4]. Em relação ao leite e seus derivados, os cuidados higiênicos para evitar a contaminação devem ser iniciados desde a ordenha e continuados até a obtenção do produto final. Diversos microrganismos patogênicos podem ser encontrados contaminando o leite, dentre eles podem-se destacar Escherichia coli e $L$. monocytogenes [33]. 
$\mathrm{Na}$ microbiologia de alimentos vem sendo estudada, nos últimos anos, a presença de Listeria spp. com predominância da espécie $L$. monocytogenes, assim como a diversidade de espécies do gênero. Contribuem para este estudo, um maior conhecimento destes patógenos nos seus aspectos epidemiológicos, clínicos e laboratoriais através do desenvolvimento de meios de cultura mais seletivos que favoreceram seu isolamento e identificação $[11,16]$. De fato, sua detecção ficou bastante facilitada em alimentos com a limitação do desenvolvimento da microbiota acompanhante [7, 17], possibilitando a utilização de procedimentos que agilizaram o diagnóstico e facilitaram seu rastreamento ambiental.

Listeria spp. é um cocobacilo Gram-positivo, nãoesporulado, não-produtor de ácidos, aeróbio e anaeróbio facultativo, de ampla distribuição ambiental, com caráter ubiquitário, tendo sido isolado de águas superficiais, de esgotos domésticos, águas residuárias de indústrias de laticínios e de abatedouros, de solos, de insetos, de adubo orgânico e em fezes de animais e inclusive de humanos [19, 23, 26]. Pode também ser isolada em diversos produtos alimentícios sejam crus ou após tratamentos térmicos ou químicos [10, 12, 31, 35].

L. monocytogenes é patogênica para o homem e diversos animais, e sua ampla distribuição ambiental, igual às outras espécies, é favorecida pela sua capacidade de se desenvolver entre $0^{\circ} \mathrm{C}$ e $44^{\circ} \mathrm{C}$ e, embora sua faixa ótima seja entre $30^{\circ} \mathrm{C}$ e $37^{\circ} \mathrm{C}$, pode sobreviver em alimentos congelados. Tolera $\mathrm{pH}$ extremos de 5 e 9, baixa atividade da água e concentrações de $\mathrm{NaCl}$ de $10 \%$ e até superiores. Este conjunto de características faz com que Listeria spp. e L. monocytogenes, em especial, sejam um patógeno emergente de grande interesse na área de alimentos e explica o destaque que estes microrganismos vêm ocupando nos últimos anos no controle de qualidade na indústria de alimentos, visto as dificuldades de sua eliminação, assim como, a possibilidade de causar uma doença grave no consumidor [20, 27, 30, 31, 37].

A literatura é abundante em relatos da presença de Listeria spp. em alimentos, seja nas matérias-primas, durante a produção e/ou durante o processamento ou nos produtos já acabados em exposição nas prateleiras $[10,31]$.

No Brasil, existem vários registros da presença de L. monocytogenes em leite e derivados [6, 13, 24, 38, 42]. Dentre eles, SILVA, HOFER, TIBANA [39], pesquisando a contaminação em queijos produzidos no Rio de Janeiro, encontraram $19,6 \%$ das amostras positivas para L. monocytogenes e, ainda, as mesmas amostras foram também positivas para $L$. innocua e $L$. grayi. A grande freqüência de casos de listeriose, veiculada por queijos, evidencia a importância desse alimento e de outros derivados do leite na cadeia epidemiológica de transmissão de Listeria spp. [35].

Curiosamente, a Portaria $n^{\circ} 451$ da Secretaria de Vigilância Sanitária do Ministério da Saúde de 19 de setembro de 1997, que estabelecia os padrões micro- biológicos para produtos alimentícios expostos à venda, não apresentava, na época que este trabalho foi realizado, nenhuma referência para valores de $L$. monocytogenes isoladas de leite [2]. Entretanto, nos Estados Unidos, a USDA (1987) fixou uma tolerância zero para esta bactéria em suprimentos alimentícios de um modo geral $[15,18]$.

A falta de notificações de casos de listeriose no Brasil, e, em particular, os associados a alimentos na região Nordeste tornam interessante a pesquisa de Listeria spp., comparando-se sua presença com a qualidade sanitária do produto, usando-se como indicadores os coliformes totais e os coliformes fecais.

O objetivo deste trabalho é investigar a qualidade microbiológica e em particular a presença de Listeria spp. no leite cru (in natura) e ao longo da linha de produção do leite pasteurizado de uma Usina de Laticínios no Estado da Paraíba, visando detectar as condições microbiológicas do produto pronto para consumo.

\section{2 - MATERIAIS E MÉTODOS}

\section{1 - Coleta das amostras de leite}

Foram analisadas 75 amostras de leite, sendo 45 de leite cru (15 provenientes do município de SousaPB, 15 do município de Campina Grande-PB, 15 do município de Garanhuns-PE) e 30 de leite pasteurizado tipo $C$ (15 coletadas na saída do pasteurizador e 15 ensacadas e prontas para comercialização). As amostras foram coletadas com freqüência quinzenal, no período de março a agosto de 1998. Em cada amostragem foram coletados ao todo 5 (cinco) litros de leite, sendo 1 (um) litro de cada tipo, utilizando-se recipientes estéreis para as amostras de leite cru e recém-pasteurizado. As amostras de leite pasteurizado, já ensacadas, foram coletadas aleatoriamente dentro da câmara refrigerada de estocagem da própria usina. $\mathrm{O}$ volume das amostras utilizadas na amostragem foi coletado independente do volume de produção da usina, pois este variava de acordo com a quantidade de leite fornecido diariamente pelos produtores. As amostras foram transportadas ao laboratório sob refrigeração, em caixa isotérmica, e analisadas em um período de aproximadamente uma hora após a coleta.

\section{2. - Pesquisa de Listeria}

Foi investigada segundo a metodologia descrita pela Food and Drug Administration-FDA, citada por ROCOURT, SCHRETTENBRUNNER, SEELIGER [34]. A técnica usada foi a seguinte: homogeneização da amostra de leite por agitação e inoculação de $25 \mathrm{~mL}$ da mesma em $225 \mathrm{~mL}$ de Caldo Enriquecido para Listeria (Caldo LEB da BIOBRÁS), adicionado de A.N.C. (Acriflavina, Ácido Nalidixico, Cicloheximida), seguido de incubação a $30^{\circ} \mathrm{C}\left( \pm 1^{\circ} \mathrm{C}\right) / 24-48$ horas. Posteriormente, após agitação do Caldo LEB, transferiu-se $0,1 \mathrm{~mL}$ deste para placa de Petri contendo Ágar Oxford (BIOBRÁS) adicionado de C.C.C.A.F (Cloreto de Lítio; Acriflavina, Fosfomicina, Colistina, Cicloheximida, Cefotetan) e o sistema 
indicador (Esculina e Citrato Férrico Amoniacal). As placas foram incubadas a $35^{\circ} \mathrm{C} / 24-48$ horas. Todas as análises foram realizadas em duplicatas. As cepas suspeitas de Listeria spp., nas placas de ágar seletivo para Listeria - Ágar Oxford, foram identificadas através de testes: morfo-tintoriais (coloração de Gram), motilidade, catalase, redução do nitrato a nitrito, produção de $\beta$ hemolisina e fermentação de carboidratos (glicose, ramnose, xilose e manitol). As cepas $\beta$-hemolisina positivas foram submetidas ao teste de CAMP com Staphylococcus aureus. Todas as cepas identificadas como $L$. monocytogrnes foram produtoras de $\beta$-hemolisina e positivas para os testes de CAMP, catalase, motilidade e fermentação da ramnose e glicose e apresentaramse negativas para os testes de redução do nitrato a nitrito e fermentação da xilose e manitol. Trinta destas cepas foram escolhidas aleatoriamente para serem testadas pelo sistema API Listeria - Bio Mérieux, a fim de comparar os resultados das duas metodologias. Todas as cepas analisadas apresentaram o mesmo resultado, confirmando assim a equivalência dos dois métodos.

\section{3 - Quantificação de coliformes totais (CT) pela téc- nica de tubos múltiplos - Número mais provável (NMP)}

Seguiu-se a metodologia da APHA [1], usando-se a técnica dos tubos múltiplos, com 3 tubos por diluição, para determinação do NMP de coliformes totais por $\mathrm{mL}(\mathrm{CT} / \mathrm{mL})$ de amostra, utilizando-se o meio Caldo Lactosado Verde Brilhante e Bile 2\% (VBB) com incubação a $35^{\circ} \mathrm{C}$ durante $24-48$ horas.

2.4 - Quantificação de coliformes fecais (CF) através da técnica de tubos múltiplos e identificação $E$. coli

Tubos de VBB positivos foram repicados para tubos com caldo E.coli suplemetado com 4 metil umbeliferil $\beta$-D glucoronídeo (EC-MUG), utilizado para determinar de forma simultânea, a presença de CF e E. coli. Após a incubação a $44,5^{\circ} \mathrm{C}$ durante 24 horas, registrou-se o número dos tubos com crescimento positivo para a presença de CF (turbidez e gás) e determinou-se o NMP/mL. Todos os tubos de EC-MUG com desenvolvimento em $24 \mathrm{~h}$ de incubação foram observados sob luz ultravioleta $(\lambda=365 \mathrm{~nm})$ numa cabine escura, considerando-se positivos para $E$. coli os que apresentaram fluorescência azul. Uma alça do material de cada um desses tubos, foi semeada em Ágar Eosina Azul de Metileno (EMB) para obtenção de colônias isoladas e após incubação a $37^{\circ} \mathrm{C} / 24$ horas; as colônias típicas foram submetidas a testes de triagem (Triple Sugar Iron - TSI; motilidade SIM, Citrato de Simmons, Uréia, Vermelho de Metila VM e Voges Proskauer - VP).

\section{3 - RESULTADOS E DISCUSSÃO}

A Tabela 1 mostra que das 75 amostras de leite, 42 (56,0\%) apresentaram Listeria spp., sendo mais freqüentes no leite cru (33 amostras - 73,3\%). É importante ressaltar que todos os tipos de leite analisados revelaram a presença de Listeria spp. em percentuais eleva- dos $(66,6 \%$ a $86,6 \%)$, variando com a origem do produto. Salienta-se presença de Listeria spp. em 9 (30\%) amostras de leite pasteurizado. L. monocytogenes foi isolada em $26(61,9 \%)$ das amostras de leite analisadas. Observa-se que os resultados para o leite pasteurizado e para o ensacado foram analisados em conjunto, ou seja, sem diferenciar a origem das amostras visto que, na usina, são misturadas as amostras de leite de todos os fornecedores, antes da pasteurização.

TABELA 1. Incidência de Listeria spp. e de L. monocytogenes em amostras de leite cru e pasteurizado (Campina Grande-PB)

\begin{tabular}{lccc}
\hline \hline Tipo de Amostras & $\begin{array}{c}\mathrm{N}^{\circ} \text { total de Amostras } \\
\text { Examinadas }\end{array}$ & $\begin{array}{c}\mathrm{N}^{\circ} \mathrm{e} \% \text { de Amostras } \\
\text { Positivas para Listeria spp. }\end{array}$ & $\begin{array}{c}\mathrm{N}^{\circ} \mathrm{e} \% \text { de Amostras Positivas para } \\
\text { L. monocytogenes }\end{array}$ \\
\hline I (Sousa-PB) & 15 & $10(66,6 \%)$ & $4(40,0 \%)$ \\
II (Garanhuns-PE) & 15 & $13(86,6 \%)$ & $8(61,5 \%)$ \\
III (C.Grande-PB) & 15 & $10(66.6 \%)$ & $5(50,0 \%)$ \\
Sub - total 1 & 45 & $33(73,3 \%)$ & $17(51,5 \%)$ \\
IV & 15 & $4(26,6 \%)$ & $4(100 \%)$ \\
V & 15 & $5(33,3 \%)$ & $5(100 \%)$ \\
Sub - total 2 & 30 & $9(30,0 \%)$ & $9(100 \%)$ \\
Total 1 e 2 & 75 & $42(56,0 \%)$ & $26(61,9 \%)$ \\
\hline
\end{tabular}

I, II e III = amostras de leite cru de diferentes produtores; IV= amostra de leite recém-pasteurizado; $V=$ amostra de leite ensacado.

Para uma melhor avaliação da distribuição temporal dos resultados, estes foram divididos em dois grupos, de acordo com o período de coleta (março a abril/ 1998 e maio a agosto/1998). Esta divisão foi caracterizada pelas melhorias das condições de higienização (sanitização) da usina de laticínios, executada entre fins do mês de abril e início do mês de maio em prol da ampliação de sua linha de produção, quando se trocaram os detergentes e sanitizantes utilizados por outros, específicos para a higienização de plantas de produtos láteos. Isto foi feito após dois meses de iniciada esta pesquisa.

A Figura 1 apresenta o percentual de positividade de Listeria spp. no total das amostras de leite cru, recém-pasteurizado e ensacado em cada período. A figura mostra também o percentual de amostras positivas, referente a todo o período estudado.

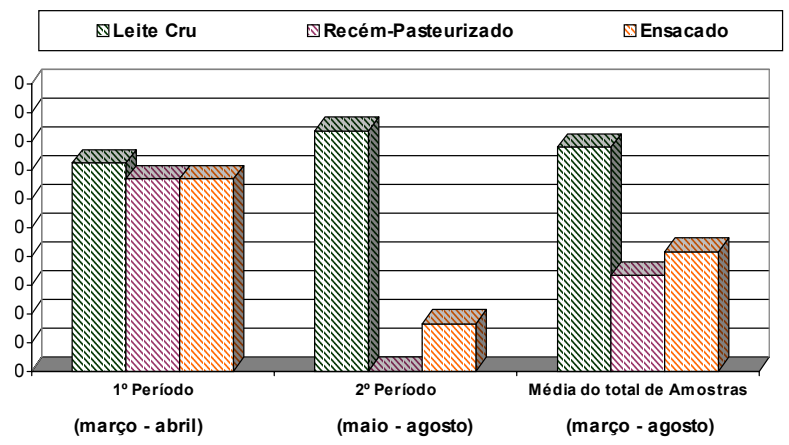

FIGURA 1. Percentual de positividade para Listeria spp. nas amostras de leite in natura (cru), recém-pasteurizado e ensacado, coletadas nos períodos de março a abril/1998 e de maio a agosto/1998

$\mathrm{Na}$ Tabela 2 observa-se que o leite in natura apresentou a maior diversidade de espécies do gênero: $L$. 
monocytogenes, L. innocua, L. ivanovii, L. welshimeri e L. grayi. Esta última foi encontrada apenas nas amostras do produtor I. Nas amostras de leite recém-pasteurizado, foram encontradas $L$. monocytogenes e nas de leite ensacado $L$. monocytogenes e $L$. innocua, sugerindo a possibilidade de contaminação pós-pasteurização.

TABELA 2. Diversidade das espécies de Listeria spp. em amostras de leite cru e pasteurizado.

\begin{tabular}{cccccccc}
\hline \hline \multirow{2}{*}{ Amostras } & \multirow{2}{*}{$\begin{array}{c}\mathbf{N}^{\circ} \text { de Amostras } \\
\text { Positivas (No de }\end{array}$} & \multicolumn{3}{c}{$\mathbf{N}^{\circ}$ (\%) de Cepas de Listeria spp. Identificadas } \\
\cline { 3 - 8 } & Cepas Identificadas) & L monocytogenes & L. innocua & L ivanovii & L. grayi & L. welshimeri \\
\hline I & $10(30)$ & $17(56,6)$ & $9(30,0)$ & $1(3,3)$ & $2(6,6)$ & $1(3,3)$ \\
II & $13(39)$ & $24(61,5)$ & $13(33,3)$ & $1(2,5)$ & $0(0,0)$ & $1(2,5)$ \\
III & $10(30)$ & $19(63,3)$ & $7(23,3)$ & $3(10,0)$ & $0(0,0)$ & $1(3,3)$ \\
IV & $4(12)$ & $12(100,0)$ & $0(0,0)$ & $0(0,0)$ & $0(0,0)$ & $0(0,0)$ \\
V & $5(15)$ & $12(80,0)$ & $3(20,0)$ & $0(0,0)$ & $0(0,0)$ & $0(0,0)$ \\
Total & $42(126)$ & $84(66,6)$ & $32(25,3)$ & $5(3,9)$ & $2(1,5)$ & $3(2,3)$ \\
\hline
\end{tabular}

I, II e III = amostras de leite cru de diferentes produtores; IV= amostra de leite recém-pasteurizado; $V=$ amostra de leite ensacado.

Observaram-se diferentes percentuais de isolamento para as várias espécies de Listeria, que foram identificadas como: L. monocytogenes $88(66,6 \%)$ das 126 cepas isoladas, L. innocua $32(25,3 \%)$, L.ivanovii 5 (3,9\%), L. welshimeri $3(2,3 \%)$ e L. grayi $2(1,5 \%)$, constatando-se a predominância de $L$. monocytogenes.

A Figura 2 mostra o percentual de diversidade e de isolamentos simultâneos das espécies de Listeria encontradas no leite cru e no pasteurizado. A maioria das amostras de leite caracterizou-se por apresentar contaminação com duas espécies ao mesmo tempo e a concomitância de ocorrência mais comum foi $L$. monocytogenes e L. innocua, tanto nas amostras de leite cru quanto nas de leite pasteurizado. De fato, esta concomitância de isolamento ocorreu principalmente com as duas espécies de maior freqüência nos diferentes tipos de leite estudados e em porcentagens menores com as outras, mais escassas. Observou-se que 47,6\% das amostras apresentaram uma única espécie sendo: L. monocytogenes $(38,1 \%)$ e $L$. innocua $(9,5 \%)$, nas demais amostras foram isoladas duas espécies.

As amostras de leite cru de todos os produtores apresentaram elevada incidência de CT, CF e E. coli, evidenciando alta contaminação da matéria-prima. Todas as amostras de leite pasteurizado analisadas nos meses de março a abril/1998 ( $1^{\circ}$ período de estudo) apresentaram-se fora dos padrões microbiológicos, que determinam para o leite tipo $\mathrm{C}$, valor máximo de $\mathrm{CT}$ de $10 \mathrm{NMP} / \mathrm{mL}$ e de CF de $2 \mathrm{NMP} / \mathrm{mL}$. Os resultados sugerem contaminação pós-pasteurização no ensacamento ou pasteurização deficiente.

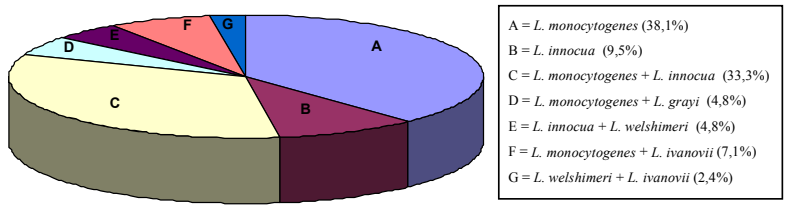

FIGURA 2. Percentual de diversidade e concomitância entre as espécies de Listeria spp. isoladas em amostras de leite cru e pasteurizado.
Após melhorias na higienização, houve uma significativa redução da contaminação. Os valores de CT e CF passaram a ser aceitáveis a partir de maio/1998, permanecendo assim durante quase todo o segundo período de estudo, onde se constatou que $11,1 \%$ das amostras recém-pasteurizadas estavam fora dos padrões para CT e 33,3\% para CF e dentre das amostras ensacadas, $22,2 \%$ estavam fora dos padrões para CT e $44,4 \%$ para CF (Tabela 3 ).

TABELA 3. Número e percentual de amostras de leite beneficiado, fora dos padrões, em relação ao período de coleta.

\begin{tabular}{|c|c|c|c|c|c|}
\hline \multirow{3}{*}{ Período de Coleta } & \multirow{3}{*}{$\begin{array}{c}\mathbf{N}^{\circ} \text { de } \\
\text { Amostras }\end{array}$} & \multicolumn{4}{|c|}{ Amostras fora dos Padrões $\left(\mathrm{N}^{\circ} \mathrm{e} \%\right)$} \\
\hline & & \multicolumn{2}{|c|}{ IV } & \multicolumn{2}{|c|}{$\mathbf{V}$} \\
\hline & & CT & CF & CT & CF \\
\hline $1^{\circ}($ março a abril/1998) & 6 & $6(100,0 \%)$ & $6(100,0 \%)$ & $6(100,0 \%)$ & $6(100,0 \%)$ \\
\hline $2^{\circ}($ maio a agosto/1998) & 9 & $1(11,1 \%)$ & $3(33,3 \%)$ & $2(22,2 \%)$ & $4(44,4 \%)$ \\
\hline
\end{tabular}

IV= amostra de leite recém-pasteurizado; V= amostra de leite ensacado

Considerando que o processo de pasteurização continuou sem alterações, as melhorias na qualidade microbiológica do leite podem ser atribuídas à higienização. Vale a pena ressaltar que o valor médio de CF nas amostras ensacadas (CF de $11,32 \mathrm{NMP} / \mathrm{mL}$ ) foi praticamente igual ao valor médio das amostras recémpasteurizadas $(10,68 \mathrm{CF} / \mathrm{mL})$, o que reforça a hipótese de que havia deficiências higiênicas no ensacamento. Também as flutuações observadas ao longo do período de amostragem para ambos indicadores foram bastante semelhantes. Comparando-se os valores de $E$. coli com os de CF em ambos os períodos, constatou-se que as flutuações foram semelhantes. As Figuras 3, 4 e 5 ilustram esse comportamento.

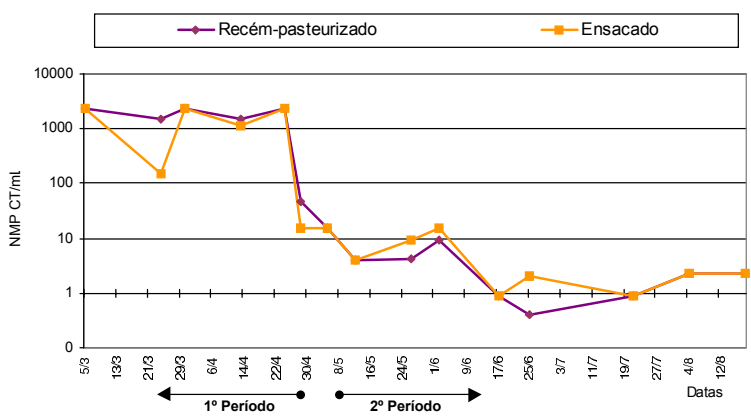

FIGURA 3. Distribuição temporal do NMP/mL de coliformes totais nas amostras de leite recém-pasteurizado e ensacado

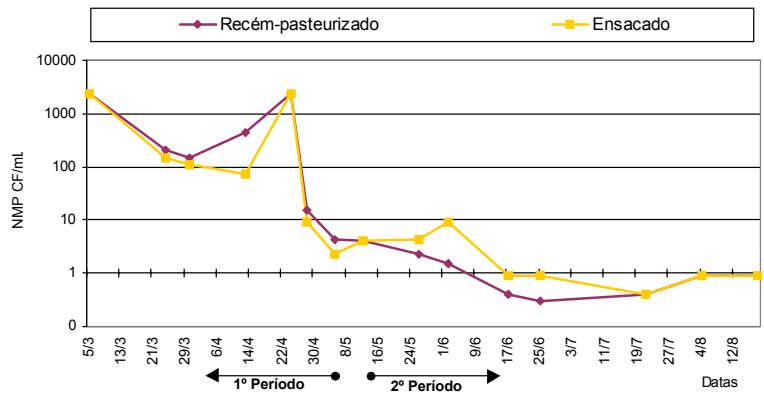

FIGURA 4. Distribuição temporal do NMP/mL de coliformes fecais nas amostras de leite recém-pasteurizado e ensacado. 


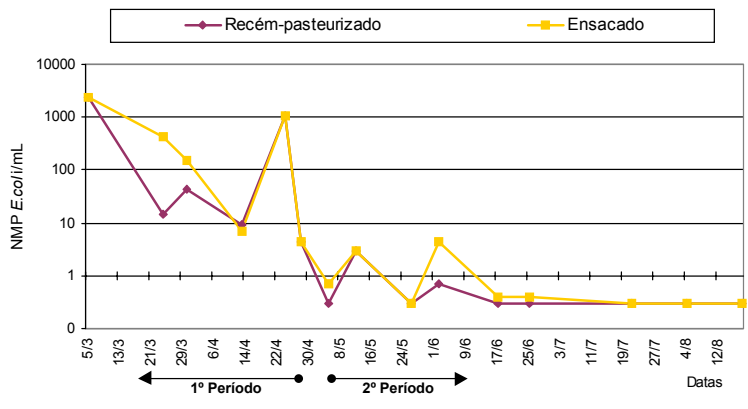

FIGURA 5. Distribuição temporal do NMP/mL de $E$. coli nas amostras de leite recém-pasteurizado e ensacado.

Na Tabela 4 pode-se observar que um elevado número de amostras de leite pasteurizado tipo $\mathrm{C}$, e de amostras ensacadas estão fora dos padrões de CT e CF. Em relação ao leite recém-pasteurizado, 7 (46,6\%) e $9(60,0 \%)$ das amostras estavam fora dos padrões, respectivamente para $\mathrm{CT}$ e $\mathrm{CF}$, enquanto no leite ensacado somente $8(53,3 \%)$ e $10(66,6 \%)$ das amostras estiveram fora dos padrões.

TABELA 4. Número e percentual de amostras de leite pasteurizado tipo $\mathrm{C}$, fora dos padrões para CT e CF, analisadas no período de março a agosto/1998.

\begin{tabular}{cccr}
\hline \multirow{2}{*}{ Amostras } & $\mathbf{N}^{\mathbf{0}}$ de Amostras Analisadas & \multicolumn{2}{c}{ Amostras Fora dos Padrões (N $\left.\mathbf{N}^{\mathbf{0}} \mathbf{0}\right)$} \\
\cline { 2 - 4 } & & $\mathbf{C T}$ & $\mathbf{C F}$ \\
\hline IV & 15 & $7(46,6 \%)$ & $9(60,0 \%)$ \\
V & 15 & $8(53,3 \%)$ & $10(66,6 \%)$ \\
Total & 30 & $15(50,0 \%)$ & $19(63,3 \%)$ \\
\hline \hline
\end{tabular}

IV= amostra de leite recém-pasteurizado; V= amostra de leite ensacado.

Neste estudo foram realizados testes qualitativos para o isolamento de Listeria spp., que permitiram determinar sua presença em amostras de leite. Métodos qualitativos são considerados adequados para atender à legislação de alguns países, que preconizam tolerância zero de Listeria em alimentos prontos para consumo [15]. A adoção do padrão contemplando a ausência de Listeria spp. em diversos países [36] visa assegurar um produto isento de risco para o consumidor, pois apesar de uma única célula de $L$. monocytogenes provavelmente não ser suficiente para causar listeriose, sua capacidade de multiplicação em condições de estocagem, mesmo sob refrigeração, faz com que sua presença no alimento coloque em risco a saúde dos consumidores mais susceptíveis (gestantes, crianças, imunodeprimidos) $[22,40]$. A completa exclusão de $L$. monocytogenes dos alimentos é irreal, porém em um futuro próximo, critérios mais convenientes devem ser estabelecidos e rigorosamente adotados [15].

Nas 75 amostras de leite (in natura e beneficiado) analisadas neste trabalho, a freqüência de Listeria spp. $(56,0 \%)$ foi elevada e dentro deste percentual $L$. monocytogenes ocorreu em $61,9 \%$, o que é considerado uma porcentagem muito alta. Embora a maioria das amostras positivas para Listeria spp. (33 amostras) fossem de leite cru, uma quantidade importante (9 amostras) foi de leite beneficiado, sendo 4 de amostras re- cém-pasteurizadas e 5 de amostras ensacadas. Estes resultados sugerem fortemente deficiências de condições higiênico-sanitárias na ordenha, no transporte ou em ambos. O isolamento em amostras pasteurizadas indica que a pasteurização não foi suficiente, ou que houve recontaminação ou, ainda, que ocorreram ambos fenômenos.

MOURA, DESTRO, FRANCO [24], investigando a incidência de Listeria spp. em 220 amostras de leite cru em São Paulo, observaram 28 (12,7\%) positivas, sendo identificadas as espécies $L$. innocua, $L$. monocytogenes, L. welshimeri e L. grayi. Em outro lote de 220 amostras de leite pasteurizado, apenas duas $(0,9 \%)$ foram positivas para Listeria e em ambas foi isolada a espécie $L$. innocua. Analisando-se os resultados desses autores e os deste estudo, os dados sugerem que é freqüente no Brasil a incidência de Listeria spp. em leite cru, independente da região geográfica. Este panorama não é exclusivo do Brasil, visto que a ocorrência de Listeria spp. em leite cru também foi registrada em outros países, como Espanha, [8]; Canadá [5, 9]; Suíça [3]; Estados Unidos da América [21, 32], Irlanda e Japão [41].

Segundo OH, MARSHALL [28], pode haver contaminação cruzada de L. monocytogenes entre os alimentos e os manipuladores, o qual pode ser, nesta pesquisa, uma das causas da contaminação do leite após pasteurizado. Manipuladores com educação sanitária deficiente poderiam ter recontaminado o produto visto que eles circulam por todos os setores da usina, desde a plataforma de recepção (do leite cru), até o ensacamento. De fato, a situação ideal seria prevenir a entrada deste patógeno no ambiente da indústria de alimentos, fazendo-se o controle do microrganismo nos pontos de origem da matéria-prima, higienizando-se cuidadosamente as plataformas de recepção do leite, sanitizando-se corretamente os equipamentos, cuidando-se da saúde, da higiene e da educação sanitária dos manipuladores e funcionários e em geral dentro da indústria, assim como manter programas de controle de pontos críticos. O conjunto de medidas devem ser orientadas a erradicar ou pelo menos a diminuir as chances de contaminação.

Uma outra possível via de contaminação do leite pasteurizado se refere às falhas no controle da temperatura do pasteurizador, que embora suficientemente altas para exercer um efeito letal sobre os coliformes, não teriam atingido a Listeria spp., uma vez que estas bactérias apresentam alta resistência térmica [10]. Entre os fatores relacionados diretamente com a pasteurização (tempo/temperatura) estão: a possibilidade de que a contaminação tenha como origem às próprias embalagens ou falta de poder germicida das lâmpadas ultravioleta (UV) utilizadas no setor de ensacamento (não havia registros quanto ao seu tempo de uso na usina, podendo estar com sua ação diminuída), ou ainda inabilidade dos manipuladores.

OH, MARSHAL [28] ressaltam a importância do tratamento da embalagem à base de ácido lático e ácido monoláurico sobre a $L$. monocytogenes, destacando que 
estes ácidos reduzem também a carga global de microrganismos. Seria interessante que esta medida fosse adotada pela indústria, através de um setor de controle de qualidade destinado ao processamento. Também deveriam ser adotadas medidas de controle sanitário do ambiente e do pessoal técnico.

Os valores de CT e CF foram bastante elevados, evidenciando a contaminação na maioria das amostras. Valores elevados de CT e CF também foram documentados por outros autores que analisaram leite tipo C tanto na região Nordeste como na região Sul do país. Estudos efetuados, em João Pessoa-PB, registraram $98 \%$ das amostras pasteurizadas e ensacadas fora dos padrões [25] e em Campinas-São Paulo, OLIVEIRA [29] constatou que $77 \%$ das amostras analisadas estavam contaminadas.

Entretanto, na legislação não há registros de valores-limites para $E$. coli que permitam avaliar o grau de contaminação do leite por este microrganismo. Devese considerar que as tendências mais modernas sobre o uso de bactérias indicadoras de contaminação fecal sugerem $E$. coli como indicador mais específico, porque seria o único membro do grupo dos CF de origem exclusivamente fecal [14].

\section{4 - CONCLUSÕES}

- $\quad$ Listeria spp. esteve presente em porcentagens elevadas de amostras de leite cru de todos os fornecedores, as quais também apresentaram elevados níveis de contaminação fecal.

- $\quad$ No leite cru houve grande diversidade de espécies de Listeria, com predominância de L. monocytogenes, seguida por $L$. innocua, e em menores porcentagens, L. ivanovii e L. grayi.

- A redução dos níveis de contaminação avaliada pela concentração de coliformes nas amostras de leite pasteurizado mostrou que melhorias na higienização/sanitização, introduzidas na linha de processamento, não foram suficientes para eliminar a presença destes microrganismos, uma vez que foram detectadas porcentagens significativas de amostras fora dos padrões para CT e CF $(11,1 \%$ das amostras recém-pasteurizadas e $22,2 \%$ das ensacadas).

- As amostras ensacadas apresentaram índices de CT e CF mais elevados que as recém-pasteurizadas, sugerindo contaminação pós-pasteurização ou falhas no armazenamento após pasteurização.

- A positividade para Listeria spp. nas amostras de leite pasteurizado, de $66,6 \%$ no primeiro período e $11,1 \%$ no segundo, demonstraram que a pasteurização não foi suficiente para eliminação da Listeria ou que a contaminação ocorreu pós-processamento. Entretanto, a diminuição das porcentagens de amostras positivas, sugere que a sanitização da usina melhorou a qualidade bacteriológica do leite beneficiado, apesar de não ter eliminado a contaminação.

\section{5 - REFERÊNCIAS BIBLIOGRÁFICAS}

[1] APHA. American Public Health Association. In: Compedium of methods for the microbiological examination of foods. Ed.:Vanderzant, C.; Splittstoesser, D. F. 3ed Washington, 1992.

[2] BRASIL. Ministério da Saúde. Secretaria de Vigilância Sanitária, Divisão Nacional de Vigilância Sanitária de Alimentos. Portaria $n^{\circ} 451$. Diário Oficial da União, Brasília, $p$. 8-15, 22 de set., 1997.

[3] BREER, C. \& SCHOPFER, K. Listeria and food. Lancet., 29: 1022, 1988.

[4] CERqueiRA, M. M. O. P. \& LEITE, M. O. Doenças Transmissíveis pelo Leite e Derivados. Cad. Esc. Téc. Vet. UFMG, 13:39-62, 1995.

[5] DAVIDSON, R.J.; SPRUNG, D. W.; PARK, C. E. \& RAYMAN, M. K. Occurence of Listeria monocytogenes, Campylobacterspp. and Yersinia enterocolitica in Manitoba raw milk. Can. J. Food Technol., 22: 70-74, 1989.

[6] DESTRO, M. T.; SERRANO, A. M. \& KABUKI, D. Y. Isolation of Listeria species from some Brazilian meat and dairy products. Food Control. 2: 110-112, 1991.

[7] DESTRO, M. T.; SERRANO, A. M.; KABUKI, D. Y. Comparison of two plating media for the isolation of Listeria $s p$ from some brazilian dairy and meat products. Rev.Microbiol, São Paulo, 23(4):256-259, 1992.

[8] DOMINGUEZ-RODRIGUEZ, L.; FERNANDEZ GARAYZABAL, J. F.; VELASQUEZ BOLAND, J. A. Isolation de microorganisms de Listeria a partir de lait cru destiné a le consommation humaine. Can. J. Microbiol., 31:938, 1985.

[9] FARBER, J. M.; SANDERS, G. W.; JOHNSTON, M. A. A survey of various foods for the presence of Listeria species. $\mathbf{J}$. Food Protect., 52 (7): 456-458, 1988.

[10] FRANCO, B. D. G. H. \& LANDGRAF, M. Microbiologia de Alimentos. Ed. Atheneu. São Paulo, 46-50. 182p, 1996.

[11] FRASER, J. A. \& SPERBER, W. H. Rapid detection of Listeria spp in food and enviromental samples by esculin hidrolydis. J. Food Protect., 51 (10): 762-765, 1988.

[12] FRAZIER, W. C. Microbiologia de los alimentos. Acribia, $4^{a}$ ed. Zaragoza, España, 681p., 1993.

[13] FURLANETTO, S. M.P.; SANTOS, M.A.A. \& HARA, C. Listeria spp. Avaliação da eficiência de quatro meios de plaqueamento no seu isoalmento. Hig. Alim., 10 (46):30-34, 1996.

[14] GARBOGGINI, I. L. A. \& GALLO, C. R. Pesquisa de Salmonella, Campylobacter, Coliformes totais e Escherichia coli em Águas de Nascentes (Bicas) em Piracicaba - SP. In: XVI Cong. Bras. Cien. Tec. Alim., Rio de Janeiro-RJ. Anais 2: 982-985, 1998.

[15] GILBERT, R. J. Zero tolerance for Listeria monocytogenes in foods - Is it necessary or realistic? In: XII International Symposium on Problems of Listeriosis. pages 351356. Perth Western. Austrália, 1995.

[16] HAYES, P.; GRAVES, L. W.; SWAMINATHAN, B.; AJELLO, W. G.; MALCOLM, G.B.; WEAVER, E.R.; RANSOM, R.; DEAVER, K.; PLIKAYTIS, B.D.; SCHUCHAT, a; WENGER, J.D.; PINNER, R. W.; BROOME, C. V. The Listeria study group. Comparisson of three seletive enrichmente methods for the isolation of Listeria monocytogenes from naturally contaminated foods. J. Food Protect., 5 (12): 952-959, 1992.

[17] JENSEN, A. Listeria monocytogenes isolation from human faecal specimens: experiments with the seletive media, Palcam and L-Palcamy. Lett. Appl. Microbiol., 16: 3235, 1993. 
[18] JONHSON, J. L.; DOYLE, M. P. \& CASSENS, R. G. Listeria monocytogenes and other Listeria spp. in meat and meat products. A review. J. Food Protect., 53: 81-91, 1990.

[19] KONEMAN, E. K; ALLEN, S. D.; JANDA, W. M.; SCHRECKENBERGER, P. C.; Jr.WINN, W. C. Color Atlas and Textbook of Diagnostic. Microbiology. $5^{\mathrm{a}}$ ed. Lippincott. NY, 1395p., 1997.

[20] LANDGRAF, M. Novos patógenos de interesse em alimentos. Bol. SBCTA, 31 (1):5-7, 1997.

[21] LUND, A. M.; ZOTTOLA, E. A \& PUSCH, D. J. Comparison of methods for isolation of Listeria from raw milk. J. Food Protect., 54: 602-606, 1991.

[22] MADDEN, J. M. Concerns Regarding the Occurrence of Listeria monocytogenes, Campylobacter jejuni, and Escherichia coli 0157:H7 in Foods Regulated by the U.S. Food and Drug Administration. Dairy, Food and Environmental Sanatization, 14 (5):262-267, 1994.

[23] MIMS, C. A.; PLAYFAIR, J. H. L.; ROITT, I. M.; WILLIAMS, R. Microbiologia Médica. $1^{\mathrm{a}}$ ed. Manole. São Paulo, 1995.

[24] MOURA, S. M.; DESTRO, M.T. \& FRANCO, B. D. G. M. Incidence of Listeria species in raw and pasteurized milk produced in São Paulo, Brazil. Int.J. Food Microbiol. 19: 229-237, 1993.

[25] NASCIMENTO, D. Contribuição ao conhecimento das condições bacteriológicas de amostras de leite tipo $\mathrm{C}$, antes e após pasteurização, vendido na cidade de João PessoaPB, 1977/78. 1982, 88p. Tese de Doutorado. Faculdade de Ciências Farmacêuticas de São Paulo.

[26] NOJIMOTO, I. T. I.; SOUZA, S. R.; VALADÃO, L. M. Ocorrência de Listeria spp em crianças da cidade de GoiâniaGoiás. RBAC, 29 (2):73-74, 1997.

[27] NOJIMOTO, I. T. I.; CENTENO, A. J.; YANAGUITA, R. M.; WATANABE, K.; KAMUMOTO, M. \& MACHADO, M. R. Susceptibilidade aos antimicrobianos de Listeria spp. isoladas de pacientes com aborto repetitivo. Rev. Bras. Anál. Clín., 26 (3): 71-74, 1994.

[28] OH, D. H. \& MARSHALL, D. L. Infuencie of packaging method, lactic and monolaurin on Listeria monocytogenes in crawfish tail meat homogenate. Food Microbiology, 12:159-163, 1995.

[29] OLIVEIRA, J. S. Qualidade microbiológica do leite. Rev. Inst. Laticínios Cândido Tostes, 31 (186): 15-20, 1976.

[30] PAPAGEORGIOU, D. K. \& MARTIN, E. H. Fate of Listeria monocytogenes during the manufacture, ripening and storage of feta cheese. J. Food Protect., 52 (2): 82-87, 1989.
[31] PEREIRA, M.L. \& ROCOURT, J. Listeria monocytogenes Uma revisão sobre aspectos taxonômicos, importância médica e em alimentos. Rev. Higien. Alim., 7 (26):5-12, 1993.

[32] REA, M. C.; COGAN, T. M. \& TOBIN, S. Incidence of pathogenic bacteria in raw milk in Ireland. J. Appl. Bact., 73:331336, 1992.

[33] RIEDEL, G. Controle Sanitário dos Alimentos. $2^{a}$ ed. São Paulo: Atheneu, 320p., 1992.

[34] ROCOURT, J.; SCHRETTENBRUNNER, A. \& SEELIGER, H. P.R..Differénciation biochimique des grupes génomiques de Listeria monocytogenes (sensu lato). Ann. Microbiol. (Institut Pasteur, Paris) 134 A: 65-71, 1983.

[35] RYSER, E. T. \& MARTH, E. H. Listeria, listeriosis and food safety. New York: Marcel Dekker. 632p., 1991

[36] SCHLECH, W. F. Overview of listeriosis. Food Control, 7 (4/ 5): 183-185, 1996.

[37] SEELIGER, H. P. R. \& JONES, D. Genus Listeria pirie 1940. In: Bergey, s Manual of Systematic Bacteriology. P. H. A. Sneath; N. S. Mair; M. E. Sharpe \& J. G. Holt (eds). Williams \& Wilkins Co., Baltimore, 1235-1245, 1989.

[38] SILVA, M. C. D. Listeria monocytogenes em queijos: ocorrência, avaliação de métodos e caracterização de cepas isoladas de queijos. 1997, 117p. (Tese de Doutoramento) - Instituto de Microbiologia - Universidade Federal do Rio de Janeiro (UFRJ).

[39] SILVA, M. C. D.; HOFER, E. \& TIBANA, A. Incidence of Listeria monocytogenes in cheese produced in Rio de Janeiro, Brazil. J. Food Protect., 61 (3): 354-356, 1998.

[40] SILVA, M. C. D.; VILARDI, T.C.C. \& TIBANA, A. Avaliação de métodos para a detecção de Listeria em queijos. Ciênc. Tecnol. Aliment., 18 (2): 150-155, 1998.

[41] YOSHIDA, T.; SATO, M.; HIRAI, K. Prevalence of Listeria species in raw milk from farm bulk tanks in Nagano prefecture. J. Vet. Med. Sci., 60 (3): 311-314, 1998.

[42] VAN DENDER, A. G. F. Listeria monocytogenes: um problema em leite e produtos lácteos. Inf Agopec., 13 (155): 19-23, 1995.

\section{6 - AGRADECIMENTOS}

À CAPES, pela bolsa PICD/UEPB concedida e pelo apoio financeiro; à Indústria de Laticínios de Campina Grande-PB, por ter gentilmente permitido a realização deste estudo nas suas instalações e, em particular, ao Prof. Dr. Ernesto Hofer, da Fundação Oswaldo Cruz, pelas valiosas sugestões na revisão deste artigo. 